\title{
L'HOMME ET LES MAMMIFÈRES DE FRANCE MÉTROPOLITAINE : ÉVOLUTION HISTORIQUE ET INTRODUCTIONS D'ESPĖCES DANS LES MILIEUX HUMIDES ET AQUATIQUES.
}

\author{
H. MAURIN, \\ avec la collaboration technique de E. GAVAZZI, pour la partie juridique.
}

Service du Patrimoine Naturel, Institut d'Ecologie et de Gestion de la Biodiversité, Muséum National d'Histoire Naturelle, 57 rue Cuvier, 75231 Paris Cedex 05, France.

\section{RÉSUMÉ}

De par ses activités diversifiées, l'homme a modifié en permanence la faune mammalienne de France métropolitaine. Responsable de certaines extinctions, il est également à l'origine de réintroductions d'espèces autochtones et d'introductions d'espèces allochtones. On note que 7 des 18 mammifères fréquentant, à des degrés divers, les milieux humides et aquatiques correspondent à des espèces introduites, la plupart du temps à partir d'échappées de captivité. En effet, la finalité principale de l'apport de ces nouvelles espèces a été, à l'origine, l'élevage pour la fourrure, même si la chasse et l'agrément ont joué un rôle non négligeable. L'introduction active des mammifères exotiques sur le territoire métropolitain a débuté à la fin du XIXème siècle et s'est poursuivie jusque dans les années 1960. Par la suite, quelques cas d'introductions, accidentelles pour l'essentiel, ont encore été enregistrés. La communauté scientifique et naturaliste a insisté depuis longtemps sur les conséquences souvent néfastes de ces apports exogènes. De fait, la réglementation s'est renforcée récemment, pour tenter d'empêcher toute nouvelle initiative incontrôlée. À l'inverse, les actions de réintroductions sont soutenues et considérées comme des outils de gestion et de conservation du patrimoine naturel.

Mots-clés : espèce introduite, mammifère, zone humide, milieu aquatique, France métropolitaine.

\section{MAN AND MAMMALS OF METROPOLITAN FRANCE : HISTORICAL EVOLUTION AND INTRODUCTIONS OF SPECIES IN WETLANDS AND AQUATIC SYSTEMS.}

\section{SUMMARY}

Due to his various activities, man has modified the mammal fauna in Metropolitan France. $\mathrm{He}$ has been responsible for some species extinctions, and has also both reintroduced native species and introduced non-native species. Among the 18 mammal species which may be found - to different extents - in wetlands and aquatic systems, 7 are introduced species ; most of them escaped from captivity. Originally, these new species were mainly imported for their fur, although some were introduced for game and leisure. The main periods of introduction of exotic mammals in the Metropolitan areas started at the end of the nineteenth century and again in the 
1960s. After this, further introductions, mostly accidental, were noticed. The scientific and naturalist communities have for a long time been concerned with the often harmful consequences of these introductions. In order to stop any further uncontrolled developments, regulations have been recently tightened, whilst reintroductions are supported and considered as tools for the management and conservation of the natural heritage.

Key-words : introduced species, mammal, wetland, aquatic system, Metropolitan France.

\section{I - INTRODUCTION}

En raison de la grande diversité de ses climats, de ses reliefs et de sa géologie, la France métropolitaine est composée d'une grande variété de milieux naturels, qui hébergent euxmêmes des biocénoses très diversifiées. Ces biocénoses évoluent constamment, dans le temps comme dans l'espace. Cette évolution est quelquefois naturelle, car les espèces peuvent étendre ou réduire leur aire de répartition sous l'action de facteurs non anthropiques, mais elle est le plus souvent due à l'action directe ou indirecte de l'homme, en particulier par l'introduction ou la réintroduction d'espèces sauvages.

Les zones humides en général, et les milieux aquatiques en particulier, constituent un pôle d'attraction certain pour de nombreuses espèces de faune, dont quelques mammifères d'assez grande taille. Ces mammifères constituent de fait des éléments très caractéristiques des biocénoses correspondantes, dans lesquelles ils jouent un rôle important. Mais la présence d'un certain nombre d'entre eux résulte d'introductions qui s'insèrent dans le contexte général des relations étroites liant l'homme et la faune sauvage.

\section{II - L'HOMME FAIT DISPARAÎTRE, RÉINTRODUIT OU INTRODUIT DES MAMMIFÈRES}

L'homme a toujours entretenu des relations privilégiées avec bon nombre d'espèces qui composent la classe des mammifères, dont il fait partie. Divers exemples le démontrent, comme les représentations des peintures rupestres de la préhistoire, les récits du bestiaire du Moyen-Âge ou, plus proche de nous et dans un autre registre, les mobilisations mondiales pour la sauvegarde des éléphants ou des baleines. Autant d'indicateurs qui illustrent bien les liens forts qui régissent, d'une manière générale, les relations " homme-mammifères". La domestication très ancienne de nombreuses espèces de ce groupe a constitué, en particulier, un contexte privilégié pour le développement de ces relations.

Mais l'harmonie n'a pas toujours été la règle entre les humains et la faune sauvage dans la mesure où, à l'origine, ces relations ont d'abord eu un caractère purement trophique : l'homme chassait les mammifères, et surtout les grands ongulés, en essayant de ne pas être la proie des gros carnivores. A l'époque historique, l'intelligence et l'acharnement développés par le genre humain, dans son rôle de prédateur ou pour éliminer définitivement des espèces qui le menaçaient ou lui faisaient concurrence, ont été fatals à certains mammifères (KEMPF, 1987 ; SAINT GIRONS, 1989 ; MAURIN et KEITH, 1994) : d'abord les " grands seigneurs " (auroch, vers le Vième siècle; bison d'Europe, vers le VIIIème siècle; cheval sauvage, vers le XVIII'̇me siècle), puis les gibiers plus modestes (pika de Corse, au XVIII ème siècle; bouquetin des Pyrénées, vers le milieu du XIXème siècle; cerf de Corse, vers 1970). Toutes ces espèces ont disparu définitivement du territoire métropolitain. Les mammifères marins ont également payé un tribut important à leur principal prédateur-concurrent (baleine des basques, vers le milieu du XIXème siècle; phoque moine, vers 1970).

Dans quelques cas, la disparition n'a été que temporaire, certaines espèces exterminées par l'homme ayant réussi à se réimplanter d'elles-mêmes de manière durable en France. Cette réapparition naturelle s'est faite à partir des populations situées à l'étranger, dans des territoires proches des frontières (loup, vers 1992) ou du littoral national (phoque gris, vers 1960 ; phoque veau marin, vers 1991). 
Au XXème siècle, l'homme a fini par prendre conscience de la nécessité de maintenir la diversité biologique à l'échelle mondiale, afin de garantir sa survie à terme. II a, par ailleurs, reconnu l'existence du nouveau concept de " patrimoine naturel hérité de nos parents et devant être transmis à nos enfants ", avec pour corollaire la responsabilité fondamentale des Etats dans ce domaine. Cet homme moderne, soucieux de réparer parfois certains de ses excès, s'efforce donc de réintroduire certaines espèces qu'il a failli faire disparaître, volontairement ou non. Ces opérations de sauvetage doivent être considérées comme des réintroductions partielles puisqu'elles n'ont concerné, pour l'instant, que des espèces au bord de l'extinction mais qui n'avaient pas totalement disparu de France. Couplées à des mesures de protection forte, elles constituent une démarche qui est maintenant reconnue comme une véritable " action de gestion conservatoire ", développée en faveur d'éléments du patrimoine naturel qui le justifient (LECOMTE et al., 1990). Dans les faits, ces réintroductions se sont révélées être des outils efficaces pour la reconstitution des populations nationales de certaines espèces très menacées, avec pour objectif la reconquête progressive d'aires de répartition suffisantes pour le maintien d'un bon niveau des populations (castor d'Europe, mouflon de Corse, ours des Pyrénées). II en est de même dans le cas de certaines espèces ayant disparu totalement (bouquetin des Alpes) ou presque totalement (lynx d'Europe) du territoire national, pour lesquelles des opérations de réintroduction ont agi en synergie avec un phénomène de reconquête naturelle, réalisé simultanément mais indépendamment par l'espèce elle-même, à partir d'une aire d'expansion trouvant son origine dans un pays jointif de la France.

II est certain que les opérations de réintroduction d'espèces autochtones, souvent médiatisées, frappent les esprits et soulèvent les passions, allant même jusqu'à engendrer des polémiques très vives. Mais il faut être conscient du fait que, sur le terrain, leur impact sur les biocénoses et écosystèmes en place est sans commune mesure avec les perturbations souvent générées par l'introduction d'espèces allochtones (MOUTOU, 1997).

\section{III - BILAN DE LA FAUNE MAMMALIENNE ACTUELLE}

Avant de présenter les espèces introduites, il est nécessaire d'avoir à l'esprit l'importance de l'ensemble du groupe des mammifères dans le monde vivant.

Les mammifères des départements d'outre-mer, dont l'impact sur les milieux aquatiques a été très peu étudié, ne seront pas traités dans notre propos.

\section{A - Nombre d'espèces dans le monde, en Europe et en France}

A l'échelle mondiale, les mammifères constituent un groupe important. Ce groupe joue en effet un rôle essentiel dans les chaînes trophiques et les écosystèmes, bien que quantitativement très modeste avec seulement 4800 espèces (WILSON et REEDER, 1993), soit $0,3 \%$ des 1400000 espèces de faune et de flore décrites par la communauté scientifique internationale (WILSON et PETER, 1988). L'intégralité de la faune mammalienne qui peuple la biosphère, sur terre et dans les eaux marines, peut être considérée comme pratiquement connue, les descriptions d'espèces nouvelles étant devenues rares.

L'Europe héberge quant à elle 212 espèces (BEAUFORT, 1990), soit à peu près $4 \%$ des mammifères du monde. Un peu plus de la moitié de ces espèces, soit 113, est présente de manière régulière en France métropolitaine. Ce chiffre comprend les mammifères marins mais exclut les espèces marines accidentelles, les espèces allochtones parfois acclimatées mais non naturalisées, ainsi que les espèces introduites de manière très localisée ou conservées ex situ (FAYARD et al., 1984 ; DUQUET et MAURIN, 1992). Les mammifères représentent, par ailleurs, $18 \%$ des 611 espèces de vertébrés de France métropolitaine (hors poissons marins).

La répartition des espèces françaises par Ordre (figure 1) met en évidence la prédominance de deux groupes : les chiroptères ( $28 \%$ du total des espèces) et les rongeurs ( $25 \%$ du total des espèces). Viennent ensuite, à égalité, les carnivores, les insectivores et les cétacés (12\% du total des espèces). Enfin, les artiodactyles $(7 \%$ du total des espèces) précèdent les lagomorphes ( $4 \%$ du total des espèces). 
Les mammifères qui fréquentent les milieux humides et aquatiques d'eau douce peuvent être répartis grossièrement en trois groupes, en fonction de leur degré d'utilisation de l'eau (MOUTOU et BOUCHARDY, 1992 ; SAINT GIRONS et al., 1993). Le premier rassemble les espèces dont la biologie et l'écologie intègrent totalement les milieux dulçaquicoles, et dont elles ne peuvent se passer ; on peut ainsi les définir comme des " utilisateurs de l'eau à temps plein ". De la même façon, le second groupe concerne les "utilisateurs de l'eau à temps partiel ", qui exploitent régulièrement les milieux aquatiques et humides sans que leur survie en dépende, et qui peuvent donc s'en passer à l'occasion. Un certain nombre d'autres espèces, qui fréquentent occasionnellement des habitats humides ou qui ont des populations encore très limitées géographiquement, le plus souvent du fait d'une introduction en France assez récente, sont rassemblées dans un troisième groupe. Au total, ces trois catégories couvrent trois insectivores, deux chiroptères, six carnivores, six rongeurs et un artiodactyle, soit 18 espèces ou $16 \%$ de la faune mammalogique française. Le détail de ces espèces est fourni dans les trois paragraphes suivants.

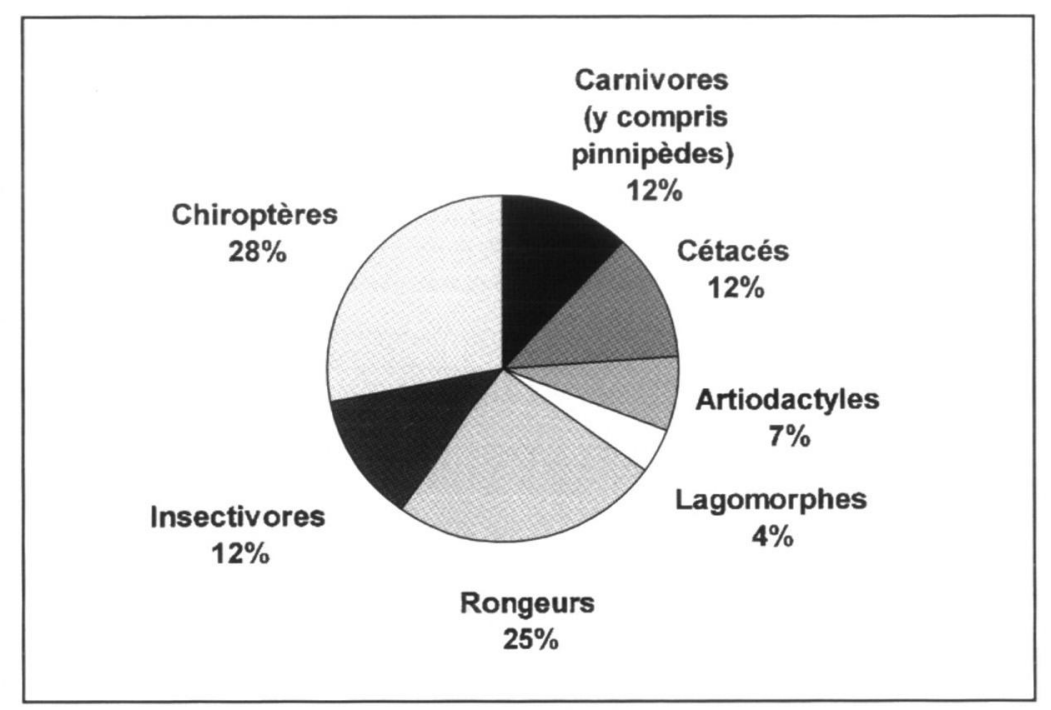

\section{Figure 1}

Répartition par Ordre (en \%) des espèces de mammifères continentaux et marins de France métropolitaine.

\section{Figure 1}

Distribution by Order $(\%)$ of continental and marine mammal species in Metropolitan France.

\section{B - Les « utilisateurs de l'eau à temps plein " \\ 1 - Les insectivores}

Trois petits insectivores discrets et peu connus sont à citer en premier lieu, même si leur existence et les conséquences de leurs activités ne constituent pas une préoccupation majeure pour l'homme (SAINT GIRONS, 1994). Ce sont d'une part deux musaraignes ou crossopes, vivant à proximité des cours d'eau (longueur tête + corps : 6 à $9 \mathrm{~cm}$; poids : 10 à $22 \mathrm{~g}$ ) : la crossope aquatique (Neomys fodiens), que l'on rencontre partout en France (sauf en Corse), et la crossope de Miller (Neomys anomalus), qui vit plutôt en altitude. C'est également le desman des Pyrénées (longueur tête + corps : 12 à $16 \mathrm{~cm}$; poids : 50 à $80 \mathrm{~g}$ ), espèce endémique inféodée aux torrents pyrénéens français et espagnols, décrite tardivement (1810). 


\section{2 - Les carnivores}

Trois carnivores, de taille nettement plus importante, sont concernés : le premier, la loutre d'Europe ou européenne (Lutra lutra), est le plus gros mustélidé aquatique de France (longueur tête + corps : 60 à $80 \mathrm{~cm}$; poids : 8 à $12 \mathrm{~kg}$ ). Présente autrefois dans les rivières, lacs et marais de tout le pays, la loutre d'Europe a vu ses populations très fortement diminuer, à la suite de piégeages et de destructions intensifs (figure 2). Elle ne subsiste plus de manière significative qu'en deux endroits : d'abord tout le long du littoral atlantique où elle continue à régresser malgré la protection complète dont elle fait l'objet. Ensuite dans le Massif Central où la situation semble un peu plus favorable, avec une amorce récente de reconquête de l'aire d'origine (ROSOUX et al., 1996).

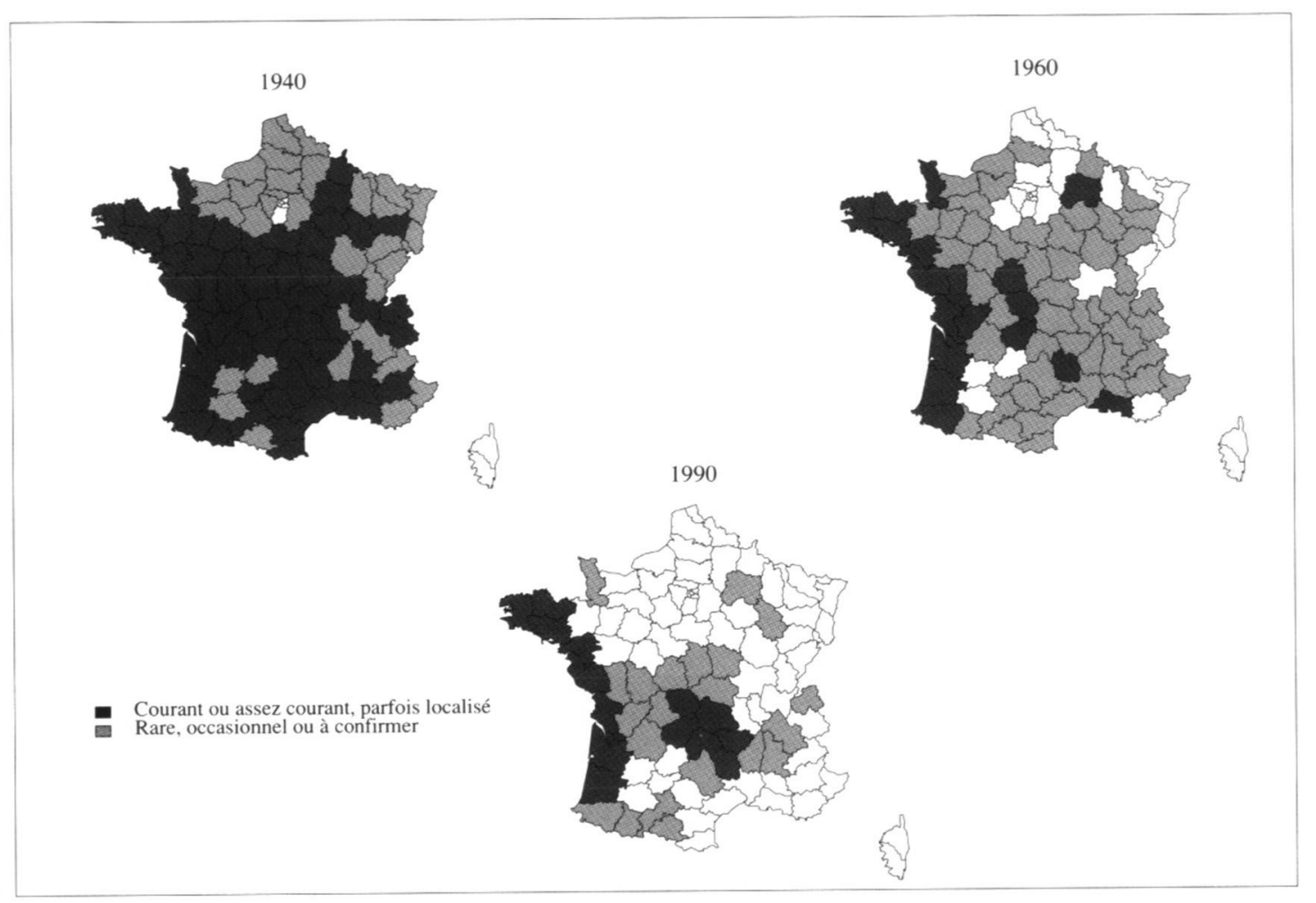

Figure 2

Répartition historique de la loutre d'Europe (Lutra lutra) en France métropolitaine.

Figure 2

Historical distribution of the common otter in Metropolitan France.

Deux espèces très voisines constituent les autres représentants de l'ordre des carnivores (CAMBY et MAIZERET, 1990) : le plus petit d'entre eux (longueur tête + corps : 30 à $40 \mathrm{~cm}$; poids : 0,4 à $1 \mathrm{~kg}$ ), le vison d'Europe ou européen (Mustela lutreola), est une espèce en très forte régression sur l'ensemble de son aire d'origine en Europe. En France, elle subsiste uniquement dans quelques départements du littoral ouest et sud-ouest. Sa régression trouve probablement, mais en partie seulement, son origine dans la concurrence qu'exerce sur elle le vison d'Amérique ou américain (Mustela vison), plus gros (longueur tête + corps : 40 à $50 \mathrm{~cm}$; poids : 1,2 à 2,2 $\mathrm{kg}$ ) et qui fréquente (semble-t-il) les mêmes habitats humides (bordures des cours d'eau et des étangs) des départements de l'ouest de la France (MAIZERET et al., 1996). Le vison d'Amérique, actuellement en pleine expansion, a été élevé au début du siècle pour sa fourrure. Echappé d'élevages, il s'est acclimaté et naturalisé rapidement sous forme de populations dynamiques, en particulier en Bretagne (mais aussi, çà et là en France), où il a sans doute éliminé le vison d’Europe. II peut donc être considéré comme une espèce introduite par l'homme. 


\section{3 - Les rongeurs}

Quatre rongeurs aquatiques, ou semi-aquatiques, complètent ce premier groupe. Le plus imposant d'entre eux (longueur tête + corps : $80 \mathrm{~cm}$ à $1 \mathrm{~m}$; poids : de 12 à $40 \mathrm{~kg}$ ), le castor d'Europe ou européen (Castor fiber), était présent partout en France autrefois, essentiellement le long des cours d'eau et des plans d'eau boisés. Ce très gros rongeur a failli disparaître, en raison des captures et des destructions massives dont il a fait l'objet. Le castor d'Europe a dû sa survie à une double action conjuguée : d'une part l'effet bénéfique de sa mise en protection dès 1905, et d'autre part les résultats favorables de diverses opérations de réintroduction (ROULAND, 1992). Ces deux facteurs ont agi en synergie et lui ont permis de reconquérir une partie de son aire d'origine, à la fois à partir de la basse vallée du Rhône où il subsistait encore (figure 3) et, dans le même temps, à partir des sites de réintroduction situés dans divers autres bassins (Loire, en particulier). Un de ses proches parents, le castor du Canada ou canadien (Castor canadensis), a été introduit à titre d'agrément en 1975 dans l'Yonne (lac du Bourdon) où il a fait souche. Mais son implantation a été volontairement interrompue par le Ministère de l'Environnement, avec l'aide des associations locales de protection de la nature, dans la mesure où son expansion aurait pu être préjudiciable à terme aux efforts de réintroduction du castor d'Europe sur la Loire (MOUTOU, 1997).

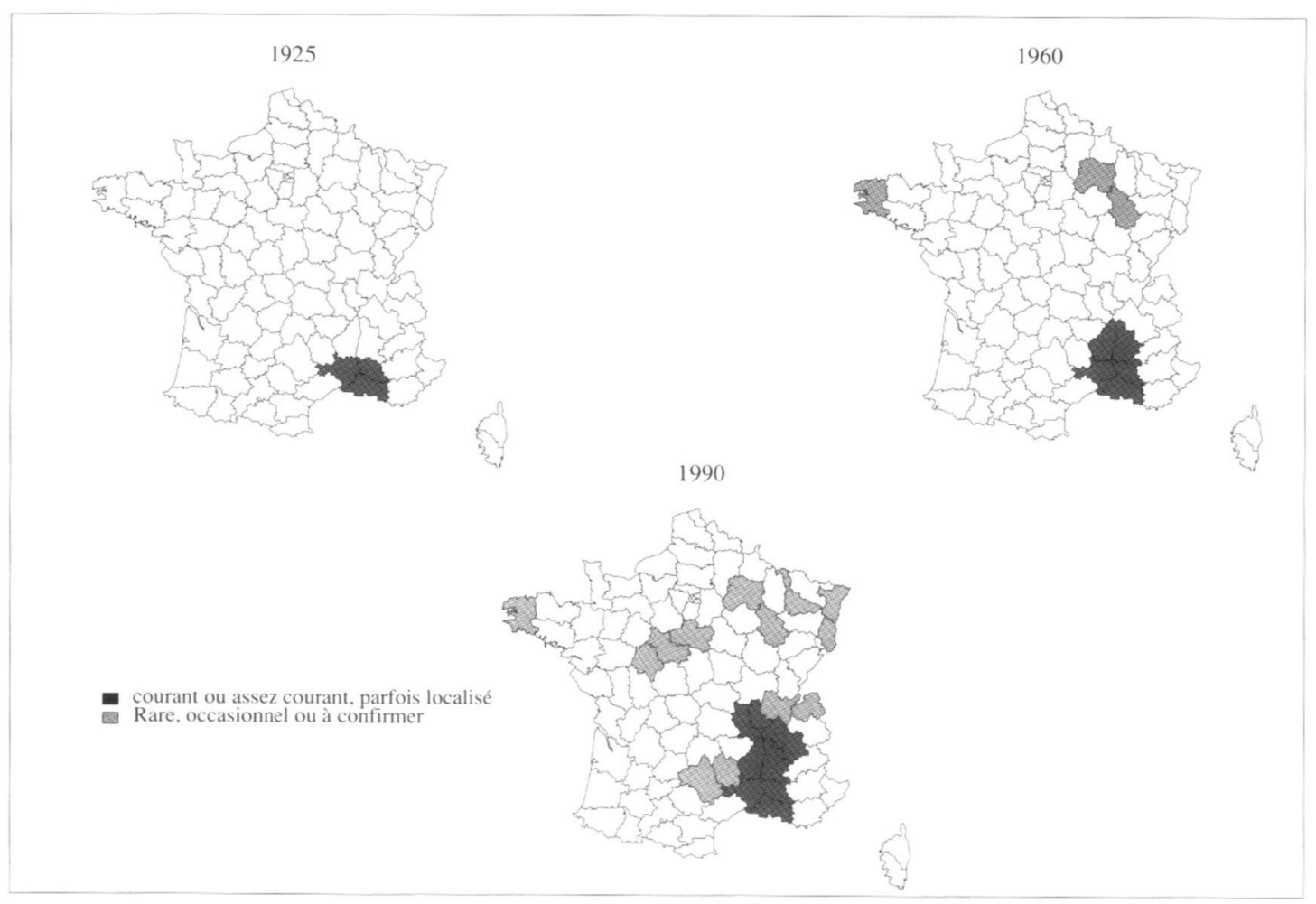

Figure 3

Répartition historique du castor d'Europe (Castor fiber) en France métropolitaine.

Figure 3

Historical distribution of the European beaver in Metropolitan France. 


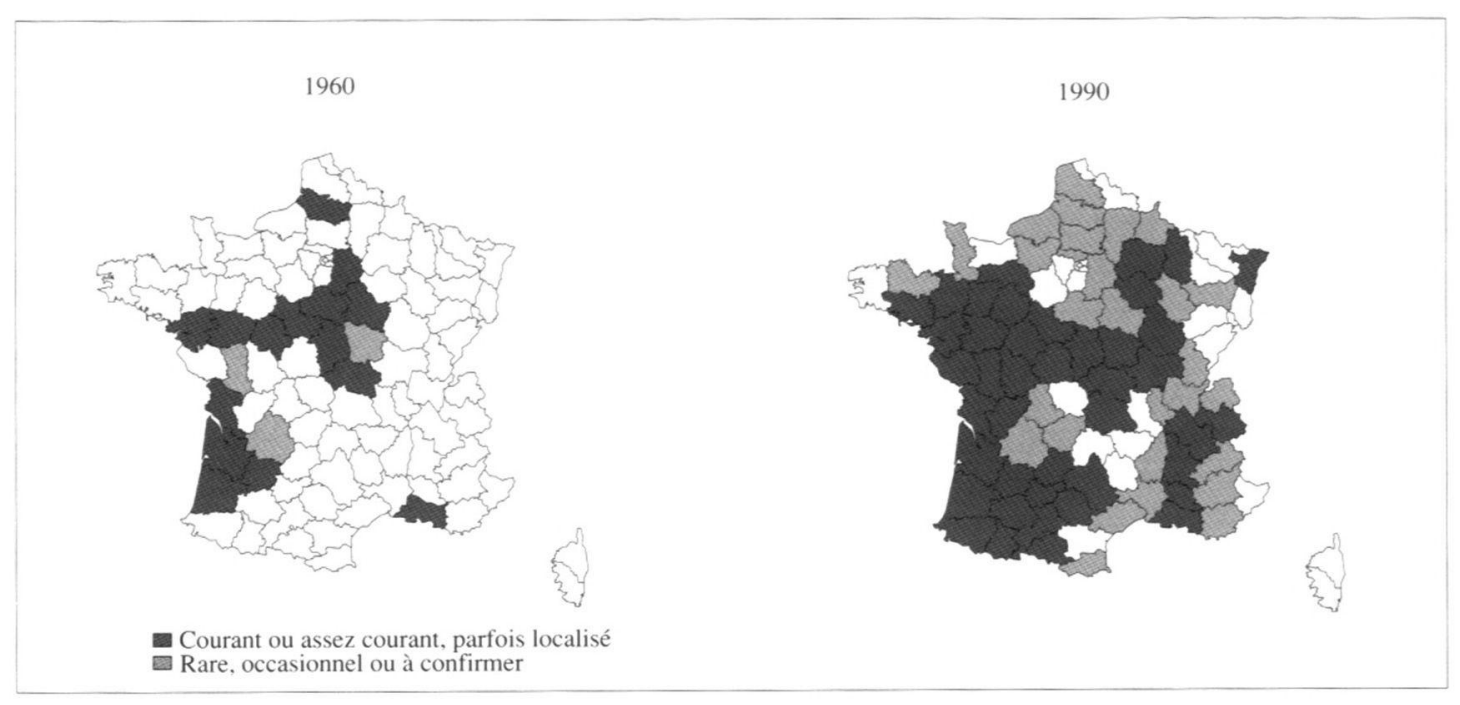

Figure 4

Répartition historique du ragondin (Myocastor coypus) en France métropolitaine.

Figure 4

Historical distribution of the coypu in Metropolitan France.

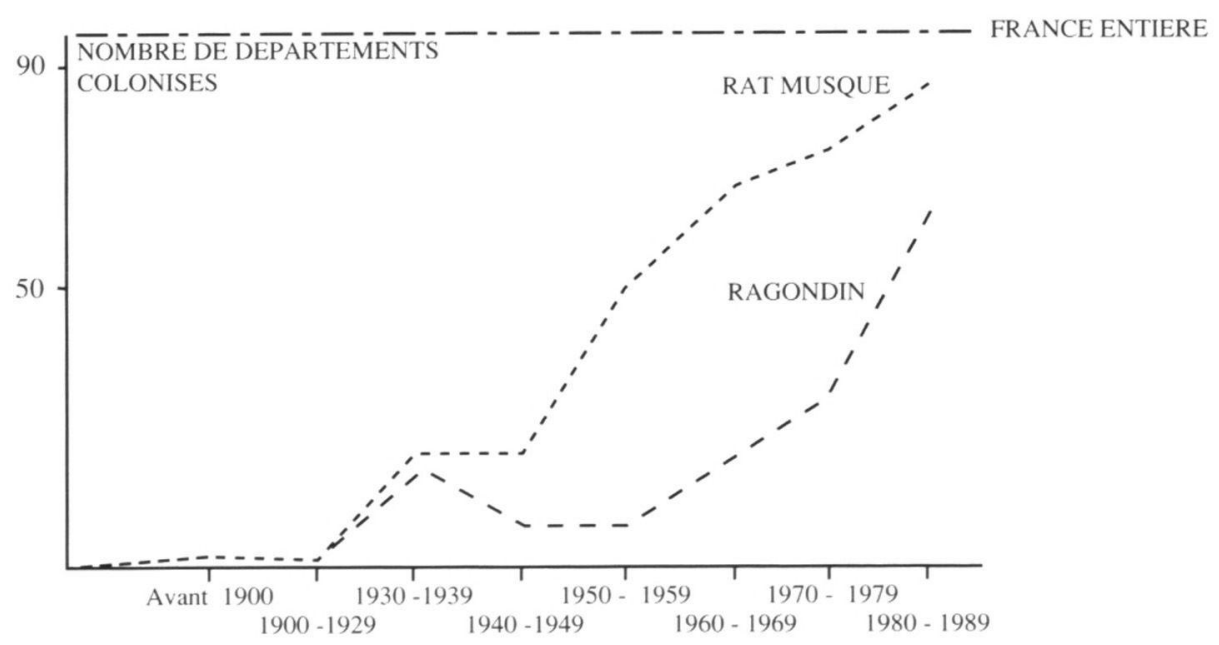

\section{Figure 5}

Courbe d'évolution comparée de la colonisation de la France métropolitaine par le rat musqué (Ondatra zibethicus) et le ragondin (Myocastor coypus).

Figure 5

Compared colonization of Metropolitan France by the musk rat and the coypu. 
Deux autres gros rongeurs non indigènes sont fortement liés aux milieux humides, et plus particulièrement aux plans d'eau, canaux et rivières à cours lent (DUQUET et MAURIN, 1992). Le ragondin (Myocastor coypus), élevé pour sa fourrure dès la fin du XIXème siècle, ressemble à un petit castor (longueur tête + corps : 40 à $60 \mathrm{~cm}$; poids : 5 à $10 \mathrm{~kg}$ ). Echappé de captivité, ou introduit volontairement dans certains cas (voir paragraphe IV-B), il a colonisé progressivement, pratiquement l'ensemble du territoire national (figures 4 et 5). En l'absence de prédateurs, il n'a été gêné dans son expansion que par les hivers froids. Le rat musqué (Ondatra zibethicus) est de taille plus modeste (longueur tête + corps : 30 à $40 \mathrm{~cm}$; poids : 1 à $2 \mathrm{~kg}$ ). Comme le ragondin, le rat musqué s'est naturalisé, lui aussi, à partir d'individus échappés d'élevages pour la fourrure, mais un peu plus tard. II a cependant colonisé l'ensemble du territoire national, beaucoup plus rapidement et régulièrement que le ragondin (figures 5 et 6 ). Ces deux espèces introduites font parfois l'objet de campagnes de destruction importantes et coûteuses, en raison des déprédations qu'elles commettent à la fois sur les cultures et dans les berges et digues (MOUTOU, 1997).

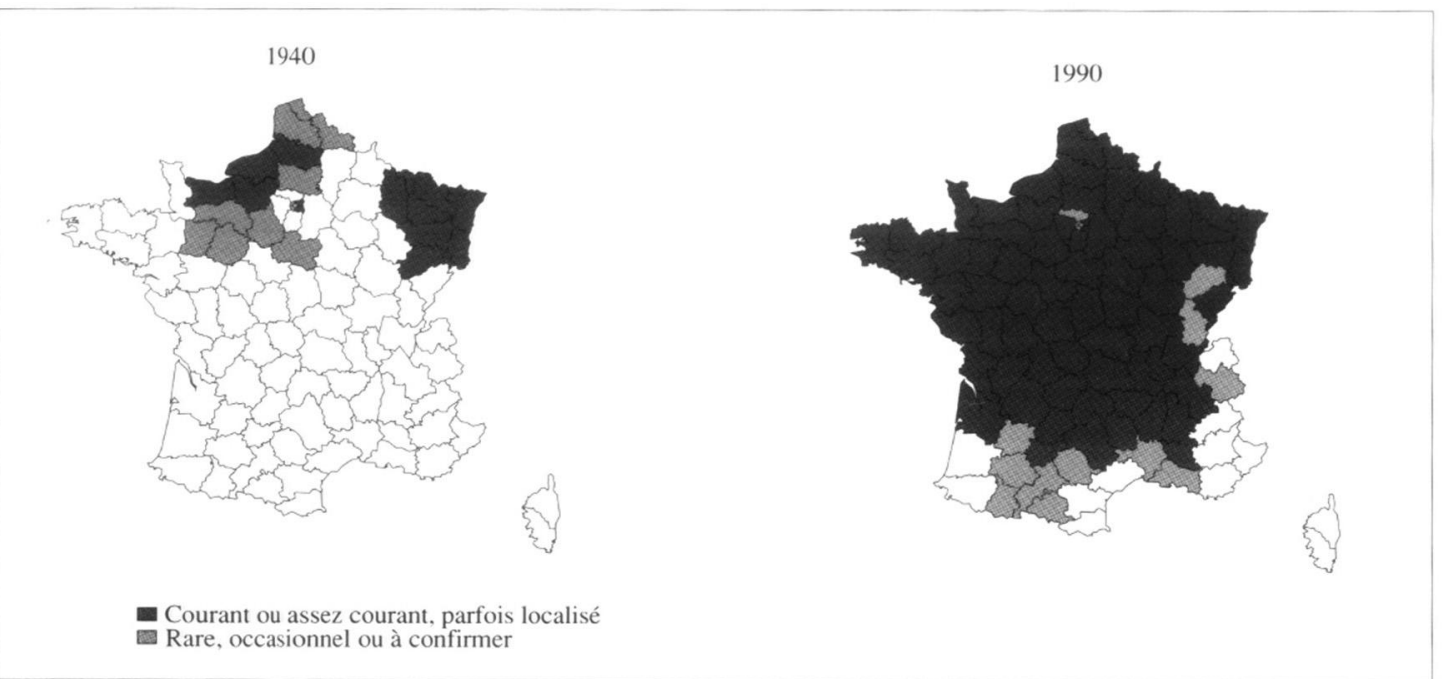

\section{Figure 6}

Répartition historique du rat musqué (Ondatra zibethicus) en France métropolitaine.

\section{Figure 6}

Historical distribution of the musk rat in Metropolitan France.

Le quatrième rongeur de ce groupe est une espèce autochtone, nettement plus modeste (longueur tête + corps : 17 à $22 \mathrm{~cm}$; poids : 150 à $280 \mathrm{~g}$ ) : le campagnol amphibie ou rat d'eau (Arvicola sapidus). Absent uniquement dans l'extrême nord et l'est de la France, il fréquente les cours d'eau lents et les étangs. Le campagnol amphibie semble actuellement se raréfier localement, peut-être du fait de la concurrence exercée sur lui par le ragondin et le rat musqué, et sûrement à cause des campagnes de piégeage et d'empoisonnement visant ces derniers (SAINT GIRONS, 1994).

Les " utilisateurs de l'eau à temps plein " correspondent donc à 10 espèces, dont 3 insectivores de petite taille, et surtout 3 carnivores et 4 rongeurs de taille nettement plus importante.

\section{C - Les “ utilisateurs de l'eau à temps partiel »}

Trois espèces, issues d'Ordres différents, utilisent régulièrement les milieux humides ou aquatiques, sans y être totalement inféodées (DUQUET et MAURIN, 1992) : 
- Un chiroptère, le vespertilion de Daubenton (Myotis daubentoni), présent partout en France. On ne sait pas grand-chose de la biologie et de l'écologie de cette chauve-souris, sinon qu'elle chasse les insectes aquatiques adultes qui émergent à la surface de l'eau et qu'elle se nourrit parfois de petits crustacés et d'alevins.

- Un carnivore, le putois (Mustela putorius), morphologiquement très proche du vison d'Europe et présent partout en France dans presque tous les milieux naturels, et en particulier dans les zones humides qu'il semble beaucoup apprécier. Faisant parfois l'objet de destructions par l'homme, dont il fréquente souvent les habitats, le putois est parfois devenu rare localement.

- Un rongeur de taille moyenne, le rat surmulot ou rat d'égout (Rattus norvegicus), qui a été introduit en Europe vers le XVIème siècle et a colonisé la France au cours du XVIIİ̀me siècle. Même s'il n'est pas particulièrement adapté au milieu aquatique, le surmulot le fréquente très souvent, nageant et plongeant avec beaucoup d'aisance. Très opportuniste, il s'est développé de façon très importante au voisinage de l'homme, en éliminant pratiquement son cousin, le rat noir (Rattus rattus). Commettant des dégâts importants, il fait très souvent l'objet de nombreuses et coûteuses campagnes de piégeage et d'empoisonnement.

\section{D - Autres espèces}

II est nécessaire de citer encore cinq espèces, soit qui fréquentent occasionnellement les milieux humides, soit qui se sont naturalisées de manière récente en France et sont restées encore très localisées:

- Un chiroptère, le vespertilion de Capaccini (Myotis capaccinii). Cette chauve-souris méditerranéenne, devenue rare, est très proche du vespertilion de Daubenton. Sa biologie est mal connue, mais on sait qu'elle recherche les milieux humides.

- Deux carnivores de taille moyenne, assez différents quant à leurs origines (DUCHENE et ARTOIS, 1988). Le raton laveur (Procyon lotor), qui a été élevé en France pour sa fourrure et a fait souche vers 1970 . Actuellement, on rencontre essentiellement cette espèce introduite dans le quart nord-est de la France où il semble rare, sauf dans l'Aisne où une petite population est bien établie. Le chien viverrin (Nyctereutes procyonoïdes), que l'on confond parfois avec le précédent bien qu'il appartienne à une famille différente, et qui a été signalé en France pour la première fois en 1979. Actuellement, il semble présent çà et là dans le quart nord-est. Il est probable que cette espèce assez discrète a gagné spontanément la France à partir des populations acclimatées en Russie dans la première moitié du XXème siècle, issues elles-mêmes d'individus échappés d'élevages pour la fourrure. Comme le surmulot, et malgré le fait que l'introduction d'origine ait eu lieu à l'étranger, il peut donc être considéré comme une espèce introduite en France. Raton laveur et chien viverrin fréquentent occasionnellement les zones humides. Ces deux espèces, qui semblent en expansion rapide, sont à surveiller tout particulièrement car elles peuvent constituer des vecteurs actifs de la rage (MOUTOU, 1997).

- Un rongeur autochtone, le minuscule rat des moissons (Micromys minutus), que l'on rencontre un peu partout en France, souvent aux abords des végétaux palustres dans lesquels il accroche son nid aérien.

- Un artiodactyle, le petit hydropote de Corée (Hydropotes inermis), introduit de manière très localisée vers 1960 dans des milieux humides du département de la Haute-Vienne.

En résumé ( 3 groupes confondus), les mammifères allochtones, naturalisés suite à une introduction et présents actuellement dans les zones humides et les milieux aquatiques de métropole, sont au nombre de $7: 3$ carnivores (le vison d'Amérique, le raton laveur et le chien viverrin); 3 rongeurs (le ragondin, le rat musqué et le rat surmulot); 1 artiodactyle (l'hydropote). 


\section{IV - QUELQUES ASPECTS DES INTRODUCTIONS DE MAMMIFÈRES}

Ce paragraphe n'abordera, dans leurs grandes lignes, que les aspects essentiels de I'historique et des finalités des introductions. II évoquera également l'évaluation des populations des espèces introduites, ainsi que leurs statuts juridiques.

La connaissance actuelle de l'évolution diachronique des mammifères en France a permis d'identifier 14 cas d'introduction, dont 7 concernent les milieux humides et aquatiques (voir paragraphe III et tableau I).

\section{Tableau I}

Finalités ayant motivé l'introduction de mammifères sauvages allochtones en France métropolitaine.

[x] : élimination pour des raisons écologiques.

(1) : introduction hors de France, à l'origine.

(2) : introduction " anthropophile " ancienne.

(3) : implantation très localisée.

Table I

Objectives of the introduction of wild allochthonous mammals in Metropolitan France.

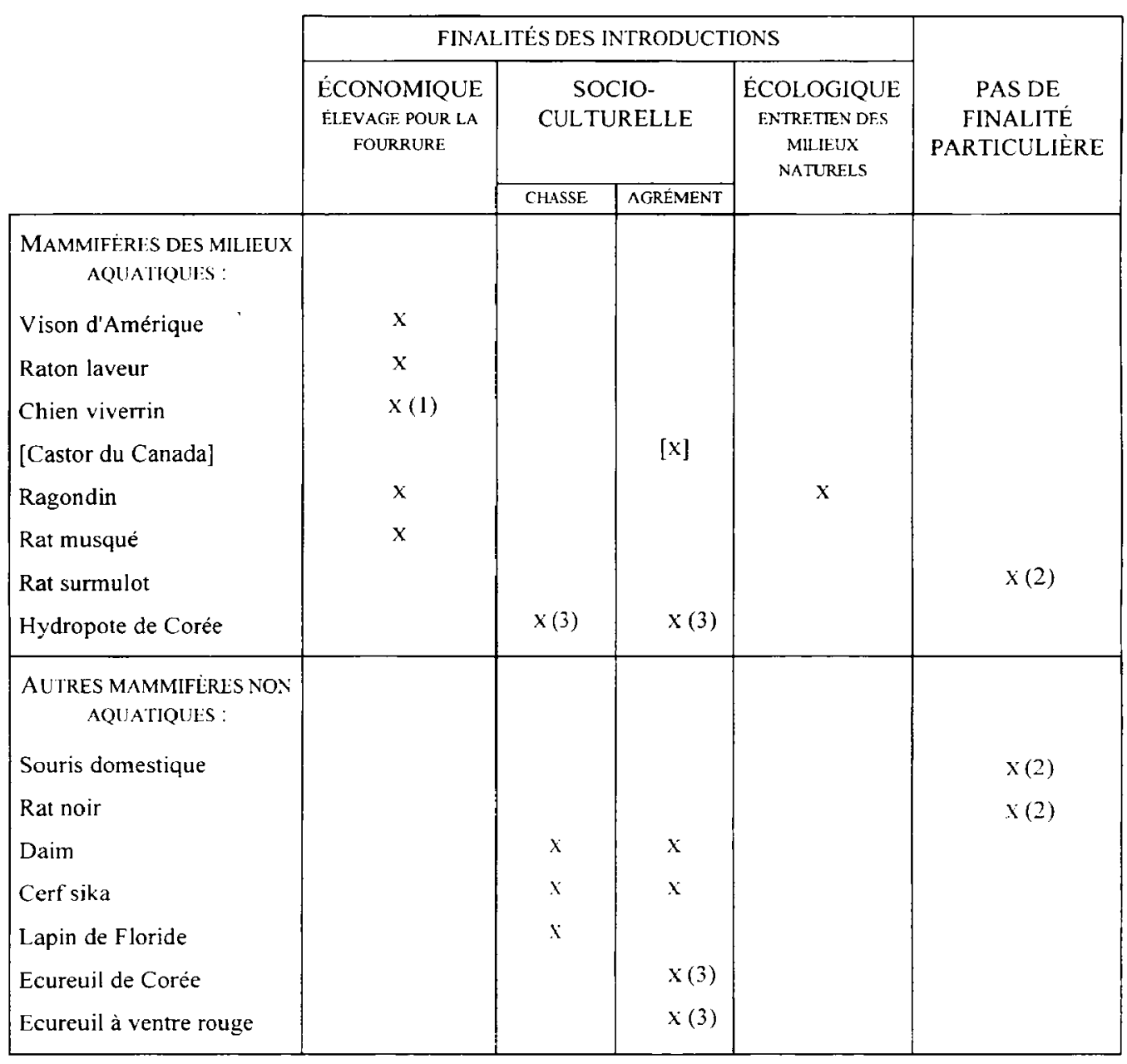




\section{A - Les introductions dans le temps}

Les premiers apports de mammifères exogènes ont débuté très tôt dans l'histoire de l'homme d'Europe occidentale, avec des introductions d'espèces anthropophiles (MAURIN et KEITH, 1994 ; SAINT GIRONS, 1994), pouvant être qualifiées " d'involontaires " (tableau I) : le rat noir (probablement vers le IVème siècle avant J.C.), la souris grise (probablement VIIème siècle avant J.C.) et, beaucoup plus près de nous, le rat surmulot (XVIIIème siècle) qui fréquente quant à lui les milieux humides ou aquatiques. Ces espèces ont suivi l'homme dans ses déplacements et ses activités (invasions, commerce...) et ont vécu à ses dépens, en étant la source d'importants dommages au plan alimentaire (réserves) ou sanitaire (peste).

Mais c'est à partir de la fin du XIXème siècle que les introductions ont été réalisées par I'homme avec des motivations particulières qu'il est possible d'identifier et de différencier (voir paragraphe B). Dans le cas des mammifères aquatiques ou semi-aquatiques, la naturalisation définitive des trois espèces dont les populations sont actuellement en expansion dans les milieux humides, n'est intervenue que dans le premier tiers du XXème siècle : ragondin (avant 1914), vison d'Amérique (après 1926) et rat musqué (après 1928). C'est plus récemment, dans les années 1960, que les deux autres espèces de mammifères exotiques fréquentant des habitats humides, le raton laveur et l'hydropote, ont fait souche. Depuis cette date, les nouvelles naturalisations n'ont concerné que les mammifères introduits pour la chasse ou l'agrément, et ne fréquentant pas spécialement les milieux humides.

Le cas du chien viverrin dont la présence en France a été constatée il y a plus d'une quinzaine d'années (1979) peut être traité un peu à part, puisqu'il s'est introduit en quelque sorte lui-même à partir de populations d'élevage, échappées à l'origine de captivité.

L'origine biogéographique des 7 espèces liées aux milieux aquatiques ou humides est variable, comme le précise MOUTOU (1997).

\section{B - Finalités des opérations d'introduction}

Qu'ils vivent à proximité de l'eau ou non, il est intéressant de classer les 14 mammifères introduits selon les finalités qui ont motivé leur importation sur le territoire naticnal (tableau I). La finalité la plus couramment identifiée est, à l'origine, une motivation d'ordre économique (LEVER, 1985; MAURIN et al., 1994). En effet 5 d'entre eux, soit un peu plus d'un tiers, fréquentant tous les milieux aquatiques ou humides, ont d'abord été élevés pour le commerce de leur fourrure (vison d'Amérique, raton laveur, chien viverrin, ragondin, rat musqué). II est à noter que les espèces de mammifères aquatiques ou semi-aquatiques ont en général une fourrure de qualité très dense, caractéristique qui explique leur sélection fréquente pour des élevages destinés à la pelleterie.

La finalité d'ordre socioculturel recouvre quant à elle deux motivations parfois difficiles à différencier, surtout dans le cas d'introductions anciennes : certaines ont été directement liées à la volonté d'améliorer localement les ressources cynégétiques, alors que d'autres ont trouvé leur fondement dans la volonté de disposer d'animaux d'agrément ou de compagnie. De fait, les espèces de mammifères dont l'acclimatation a été tentée pour la chasse, par introduction directe dans le milieu (lapin de Floride), ne sont pas spécifiques des zones humides. Il en est généralement de même des animaux d'agrément qui n'ont, quant à eux, pas été introduits directement à l'état sauvage (sauf le castor du Canada). Captifs à l'origine, ils ont souvent été placés par des particuliers dans des conditions favorables pour reconquérir à terme leur liberté, grâce à des lâchers plus ou moins volontaires ou des échappées de parcs (hydropote, cerf sika, daim) et de cages « de compagnie " (écureuil de Sibérie, écureuil à ventre rouge).

On ne recense pas, chez les mammifères, d'introductions à finalité purement écologique. Dans le seul cas du ragondin, l'argument écologique - l'espèce aurait été un " faucardeur bénévole " de la végétation aquatique (MAURICE, 1932) - a été employé lors de l'introduction, 
en plus de la motivation initiale d'ordre économique (fourrure). Mais le côté subjectif de cette affirmation, et surtout les dégâts importants dont ce mammifère s'est rendu coupable, ont rendu ce discours rapidement peu convaincant.

Dans le cas des introductions anciennes (rat noir, souris grise, rat surmulot), aucune finalité particulière ne peut être mise en évidence, hormis le fait que ces espèces étaient avant tout, mais pas uniquement, anthropophiles.

\section{C - Les niveaux de population des espèces introduites}

La connaissance de la répartition des mammifères introduits en France est variable (FAYARD et al., 1984 ; DUQUET et MAURIN, 1992 ; SAINT GIRONS et al., 1993 ; GAUTHIER et al., 1996) : bonne pour la plupart des espèces, mais encore insuffisante dans certains cas. Cette différence est due essentiellement à la rareté de certains mammifères, qui posent par ailleurs des difficultés particulières d'observation du fait d'un mode de vie le plus souvent nocturne. D'autres difficultés trouvent leur origine dans les risques de confusion qui existent dans la détermination d'espèces morphologiquement assez voisines (raton laveur et chien viverrin) ou très voisines (vison d'Europe et vison d'Amérique).

La connaissance globale des niveaux de population de chacune d'entre elles reste quant à elle nettement insuffisante. Les savoirs sont encore très partiels, même si les espèces menacées (loutre d'Europe surtout, vison d'Europe, castor d'Europe, ou même desman des Pyrénées : ROULAND, 1992 ; BERTRAND, 1993 ; GAUTHIER et al., 1996) ou celles qui sont en expansion depuis plusieurs dizaines d'années et dont la pullulation pose des problèmes à l'homme (rat musqué, ragondin : ABBAS, 1988 ; DONCASTER et MICOL, 1990 ; MICOL, 1991), ont fait l'objet d'un peu plus d'études que les autres. En effet, l'évaluation des populations nationales de n'importe quelle espèce, introduite ou non, nécessite des dénombrements dans le cadre de programmes de recherche assez lourds. Si un suivi a pu être mis en place pour certaines espèces à répartition encore restreinte (raton laveur, hydropote : LEGER et al., 1992 ; SAND et KLEIN, 1995), la connaissance exhaustive des populations sauvages devient irréalisable pour des espèces à aire plus large. Pour ces dernières, les données disponibles ne peuvent concerner que des études de terrain, partielles au plan géographique, qui fournissent des informations à valeur locale sur les densités de populations observées dans les sites étudiés. Mais l'extrapolation au plan national des chiffres obtenus reste toujours très délicate.

S'ils ne peuvent compter sur des études exhaustives proposant des dénombrements fins, les gestionnaires du patrimoine naturel ont besoin de disposer au minimum d'évaluations quantitatives qui soient représentatives. Il faut donc faire appel à d'autres sources de données, comme les résultats des programmes de destruction mis en place pour les gros rongeurs aquatiques, les données issues des études d'écoéthologie, d'écotoxicologie, ou les taux de mortalité naturelle ou accidentelle constatés chez les carnivores aquatiques menacés. Ces travaux sont en effet susceptibles de fournir des informations intéressantes, en particulier sur la capacité d'accueil du milieu pour une espèce donnée : à titre d'exemple, on a constaté qu'en 1986, 144 ragondins ont pu être abattus en six heures, dans trois communes des marais de la Sèvre Niortaise (SAINT GIRONS et al., 1993 ; GAUTHIER et al., 1996).

\section{D - Statut juridique des mammifères des milieux humides et aquatiques}

Le statut juridique des mammifères fréquentant les milieux humides et aquatiques est résumé dans le tableau II. Les dix-huit espèces peuvent être réparties dans un ou plusieurs des six grands types de statuts. Un premier groupe de huit espèces est concerné par une protection à l'échelon européen (Directive communautaire " Habitats-Faune-Flore ", Convention de Berne) et/ou une protection intégrale au niveau national. Un second groupe de sept espèces est constitué des mammifères soumis à protection partielle ou pouvant être chassés ou déclarés "nuisibles". Enfin, un troisième groupe de trois espèces concerne les rongeurs sans statut juridique particulier. 


\section{Tableau II}

Statut juridique des espèces de mammifères fréquentant régulièrement ou occasionnellement les milieux humides et aquatiques, en France métropolitaine.

(I) : espèce introduite.

(1) : directive n 92/43/CEE du Conseil du 21 mai 1992 " Habitats-Faune-Flore ", annexes II ou IV, et Convention de Berne, annexes II et III.

(2) : arrêté ministériel du 17 avril 1981, article 1er, J.0. du 19/05/81 + arrêté modificatif du 22/07/93, J.O. du 11/09/93.

(3) : arrêté ministériel du 17 avril 1981, article 2e, J.0. du 19/05/81.

(4) : arrêté ministériel du 26 juin 1987, J.0. du 20/09/87.

(5) : arrêté ministériel du 30 septembre 1988, J.0. du 02/10/88.

(6) : réglementation permanente de la Police de la chasse au niveau départemental.

(7) : fait cependant l'objet de campagnes intenses de destruction.

Table II

Legal status of mammal species which are regularly or occasionally present in wetlands and aquatic systems, in Metropolitan France.

\begin{tabular}{|c|c|c|c|c|c|c|c|}
\hline & $\begin{array}{l}\text { Protection } \\
\text { au niveau } \\
\text { européen } \\
\text { (1) }\end{array}$ & $\begin{array}{l}\text { Protection } \\
\text { nationale } \\
\text { intégrale } \\
\text { (2) }\end{array}$ & $\begin{array}{c}\text { Protection } \\
\text { nationale } \\
\text { particlle } \\
\text { (3) }\end{array}$ & $\begin{array}{c}\text { Chassable } \\
\text { au niveau } \\
\text { national } \\
\text { (4) }\end{array}$ & $\begin{array}{l}\text { Classement } \\
\text { nuisible } \\
\text { possible au } \\
\text { niveau } \\
\text { départemental } \\
\text { (5) }\end{array}$ & $\begin{array}{c}\text { Protection } \\
\text { police } \\
\text { de la chasse } \\
\text { départementale } \\
\text { (6) }\end{array}$ & $\begin{array}{c}\text { Pas de } \\
\text { statut } \\
\text { juridique } \\
\text { particulicr }\end{array}$ \\
\hline $\begin{array}{l}\text { Musaraigne aquatique } \\
\text { Musaraigne de Miller } \\
\text { Desman des Pyrénćcs }\end{array}$ & $x$ & $\begin{array}{l}x \\
x \\
x\end{array}$ & & & & & \\
\hline $\begin{array}{l}\text { Vespertilion de Daubenton } \\
\text { Vespertilion de Capaccini }\end{array}$ & $\begin{array}{l}x \\
x\end{array}$ & $\begin{array}{l}x \\
x\end{array}$ & & & & & \\
\hline $\begin{array}{l}\text { Loutre d'Europe } \\
\text { Vison d'Europe } \\
\text { Vison d'Amérique } \\
\text { Putois } \\
\text { Raton laveur } \\
\text { Chien viverrin }\end{array}$ & $\begin{array}{l}x \\
x\end{array}$ & $\begin{array}{l}x \\
x\end{array}$ & $\mathrm{X}$ & $\begin{array}{l}x \\
x \\
x \\
x\end{array}$ & $\begin{array}{l}x \\
x \\
x \\
x\end{array}$ & & \\
\hline $\begin{array}{l}\text { Castor d'Europe } \\
\text { Ragondin } \\
\text { Rat musqué } \\
\text { Rat surmulot } \\
\text { Campagnol amphibie } \\
\text { Rat des moissons }\end{array}$ & $x$ & $x$ & & $\begin{array}{l}x \\
x\end{array}$ & $\begin{array}{l}x \\
x\end{array}$ & & $\begin{array}{c}x(7) \\
x \\
x\end{array}$ \\
\hline Hydropote de Corée & & & & & & $x$ & \\
\hline
\end{tabular}


Ce tableau montre également que, mis à part l'hydropote, les six autres mammifères introduits en milieu humide sont chassables ou susceptibles d'être classés nuisibles, ou encore soumis à des campagnes de destruction en raison des dégâts qu'ils sont (ou seraient) susceptibles de commettre vis-à-vis de l'homme. Cette caractéristique commune met bien en évidence l'existence de conséquences très négatives de ces introductions (MOUTOU, 1997).

\section{V - CONCLUSION}

Au plan historique, les faunes de vertébrés ont toujours évolué sous l'action directe ou indirecte de l'homme. En particulier, les introductions d'espèces nouvelles ont modelé de façon significative la faune mammalienne fréquentant les milieux aquatiques de métropole.

Les chercheurs et naturalistes font le constat de ces évolutions et en assurent le suivi scientifique sur le terrain. Par ailleurs, les gestionnaires du patrimoine naturel (administrations, grands organismes techniques, associations, ...) ont besoin de connaître les tendances affectant les espèces " sous surveillance biologique " (introduites, en particulier) et leurs populations, afin d'orienter les politiques de gestion patrimoniale, tant au niveau local que national ou européen.

Après avoir été longtemps encouragées, les opérations d'introduction sont maintenant de plus en plus contestées par les divers protagonistes de la conservation de la nature. En particulier en raison d'un certain nombre d'expériences malheureuses, voire catastrophiques tant au plan économique qu'écologique ou sanitaire évoquées par MOUTOU (1997), la communauté scientifique a pris position dans ce sens de manière quasi unanime. A l'inverse, le monde cynégétique génère encore un certain nombre de partisans de l'introduction de nouvelles espèces susceptibles de s'acclimater, en vue d'augmenter les possibilités d'exploitation de la faune par la chasse. De fait, le renforcement récent des réglementations françaises et européennes tend à empêcher ou contrôler toute nouvelle initiative dans ce domaine (BENTATA, 1997 ; GALLAND, 1997).

La situation est totalement différente dans le cas des opérations de réintroduction, menées primitivement à l'initiative des associations de protection de la nature. Celles-ci sont maintenant considérées comme des outils de gestion et de conservation, et leur usage tend à se développer pour les espèces les plus menacées. Les réintroductions ne doivent toutefois pas être confondues avec les actions de repeuplement, menées le plus souvent au coup par coup et sans recul suffisant, particulièrement dans le cas des espèces de " petit gibier " ou du sanglier.

Il paraît de plus en plus évident qu'à l'avenir, aucun apport d'espèce allochtone ne devrait être envisagé sans qu'un certain nombre de garanties aient été prises au préalable : étude scientifique sérieuse, protocole de suivi adapté, respect d'une déontologie admise par tous, évaluation des impacts prévisibles à moyen et long terme sur les milieux et les biocénoses en place... II est impératif que ces précautions intègrent également le contexte socioéconomique et culturel local, afin d'éviter une éventuelle et parfois violente réaction de rejet de la part de certains usagers du milieu naturel concernés (éleveurs, agriculteurs, forestiers, chasseurs, pêcheurs ...).

\section{VI - REMERCIEMENTS}

L'auteur tient à remercier ses précieux collaborateurs du Service du Patrimoine Naturel de l'IEGB du MNHN et tout particulièrement, au plan scientifique : P. KEITH, P. HAFFNER et P. DASZKIEWICZ ; au plan informatique : J.F. BRULARD et H. DA COSTA; et au plan technique : P. ALEXANDRE, G. PROCIDA et L. DAUVIN.

Il tient également à insister sur le fait que l'amélioration permanente des connaissances sur les mammifères repose surtout sur l'activité bénévole des naturalistes regroupés, au plan 
associatif, au sein de la Société Française pour l'Étude et la Protection des Mammifères et de nombreuses autres associations régionales et départementales, ainsi que sur le travail des agents de l'Office National de la Chasse, de l'Office National des Forêts et des espaces protégés. Qu'ils soient tous remerciés chaleureusement.

\section{VII - BIBLIOGRAPHIE}

ABBAS A., 1988. Régime alimentaire d'un phytophage introduit, le ragondin (Myocastor coypus, Molina, 1792) dans différents types de marais aménagés. Thèse de l'Université de Rennes I, $200 \mathrm{p}$.

BEAUFORT (de) F., 1990. Statut des mammifères d'Europe. Contribution scientifique de la France pour la Commission Economique pour l'Europe. Muséum National d'Histoire Naturelle, Paris, $28+30 p$.

BENTATA V., 1997. Le statut juridique en droit interne des espèces animales liées aux milieux aquatiques continentaux métropolitains. Bull. Fr. Pêche Piscic., 344-345.

BERTRAND A., 1993. Découvrir les desmans des Pyrénées. Association des Naturalistes de l'Ariège, Clermont.

CAMBY A., MAIZERET C., 1990. Le vison d'Europe (Mustela lutreola, Linnaeus, 1761) et le vison d'Amérique (Mustela vison, Schreber, 1777). Encyclopédie des carnivores de France, vol. 13 et 14. Société Française pour l'Étude et la Protection des mammitères, Paris, $44 \mathrm{p}$.

DONCASTER P., MICOL T., 1990. Response by coypus to catastrophic events of cold and flooting. Holartic Ecology, 13, 98-104.

DUCHENE M.J., ARTOIS M., 1988. Les carnivores introduits : chien viverrin (Nyctereutes procyonoides, Gray, 1834) et raton laveur (Procyon lotor, Linnaeus, 1758). Encyclopédie des carnivores de France, vol. 4 et 5 . Société Française pour l'Étude et la Protection des Mammifères, Paris, $50 \mathrm{p}$.

DUQUET M., MAURIN H., 1992. Inventaire de la Faune de France, vertébrés et principaux invertébrés, deuxième édition 1995. Nathan et Muséum National d'Histoire Naturelle, Paris, $416 \mathrm{p}$.

FAYARD A. et al., 1984. Atlas des mammifères sauvages de France. Société Française pour l'Étude et la Protection des Mammifères, Paris, $300 \mathrm{p}$.

GALLAND J.P., 1997. Les nouvelles dispositions du code rural sur les introductions d'espèces issues de la loi du 2 février 1995 relative au renforcement de la protection de l'environnement. Bull. Fr. Pêche Piscic., 344-345.

GAUTHIER J.Y., LIBOIS R., ROSOUX R., 1996. La loutre et le vison d'Europe (Colloque SFEPM, Niort 1993). Cahiers d'Ethologie, 15 (2-3-4), 434 p.

KEMPF C., 1987. Le retour des seigneurs de nos forêts d'Europe. Sang de la terre, Paris, $218 \mathrm{p}$.

LECOMTE J., BIGAN M., BARRE V., 1990. Réintroductions et renforcement de populations animales en France. Actes de colloque, décembre 1988. Rev. Ecol. Terre et Vie, Suppl. 5, 1-119.

LEGER F., DUCHENE M.J., LIENARD P., DUMONT S., ARTOIS M., 1992. Invasion du raton laveur dans l'est de la France et risques d'apparition d'un foyer de rage sur cette espèce. In SENOTIER J.L., Introductions et réintroductions de mammifères sauvages, Colloque SFEPM, Orléans 1990, 135-144. 
LEVER C., 1985. Naturalized mammals of the world. Longman, London and New York, $487 \mathrm{p}$.

MAIZERET C., MAURIN H., MIGOT P., LAFONTAINE L., 1996. Etat davancement du programme d'étude de la répartition du vison d'Europe en France. In GAUTHIER J.Y.. LIBOIS R., ROSOUX R., La loutre et le vison d'Europe. Colloque SFEPM. Niort 1993. Cah. d'Ethol., 15 (2-3-4), 419-424.

MAURICE A., 1932. Le ragondin. Arch. Hist. Nat., 7, 234 p.

MAURIN H., KEITH P., 1994. Inventaire de la Faune menacée en France, le livre rouge. Nathan, Muséum National d'Histoire Naturelle et WWF France, Paris, $175 \mathrm{p}$.

MAURIN H., HAFFNER P., KEITH P., 1994. Bilan des introductions et réintroductions de vertébrés sauvages en France métropolitaine depuis le début du siècle. In BODSON L., Colloques d'histoire des connaissances zoologiques, tome 5, Des animaux introduits par l'homme dans la France et l'Europe, Université de Liège, 79-104.

MICOL T., 1991. Statut et gestion des populations de ragondins dans leurs pays d'introduction. Bulletin Technique d'Information $n^{\circ} 3$, Les Vertébrés ravageurs des végétaux. Ministère de l'Agriculture.

MOUTOU F., 1997. Mammifères aquatiques et semi-aquatiques introduits en France. Risques et conséquences. Bull. Fr. Pêche Piscic., 344-345.

MOUTOU F., BOUCHARDY C., 1992. Les mammifères dans leur milieu. Bordas, Paris, $256 \mathrm{p}$.

ROSOUX R., TOURNEBIZE T., MAURIN H., BOUCHARDY C., 1996. Etude sur la répartition de la loutre d'Europe (Lutra lutra L.) en France. Actualisation 1993. In GAUTHIER J.Y., LIBOIS R., ROSOUX R., La loutre et le vison d'Europe, Colloque SFEPM, Niort 1993. Cah. Ethol., 15 (2-3-4), 195-205.

ROULAND P., 1992. Essai de synthèse sur la réintroduction du castor en France et perspectives. In SENOTIER J.L., Introductions et réintroductions de mammifères sauvages, Colloque SFEPM, 1990, 33-57.

SAINT GIRONS M.C., 1989. Les Mammifères en France. Sang de la terre et la Manufacture, $250 \mathrm{p}$.

SAINT GIRONS M.C., 1994. Les mammifères. In MAURIN H. et KEITH P., Inventaire de la Faune menacée en France, le livre rouge. Nathan, Muséum National d'Histoire Naturelle et WWF France, Paris, 18-43.

SAINT GIRONS M.C., MAURIN H., ROSOUX R., KEITH P., 1993. Les mammifères d'eau douce, leur vie, leurs relations avec l'homme. Ministère de l'Environnement, Ministère de l'Agriculture et Société Française pour l'Étude et la Protection des Mammifères, Paris, $48 \mathrm{p}$.

SAND E., KLEIN F., 1995. Les populations de daim, de cerf sika et d'hydropote en France. Bull. mens. de l'Office ntnl. Chasse, 205, 32-39.

WILSON E.O., PETER F.M., 1988. Biodiversity. National Acad. Press, Washington.

WILSON D.E., REEDER D.M., 1993. Mammal species of the world, taxonomic and geographic reference (second edition). Smithsonian Institution Press, Washington and London, $1206 \mathrm{p}$. 